\title{
Dysregulation of coordinated neuronal firing patterns in striatum of freely behaving transgenic rats that model Huntington's disease
}

\author{
Benjamin R. Millera, Adam G. Walkera, Stephen C. Fowler ${ }^{\mathrm{b}}$, Stephan von Hörsten ${ }^{\mathrm{C}}$, Olaf \\ Riess $^{\mathrm{d}}$, Michael A. Johnsone ${ }^{\mathrm{e}}$, and George V. Rebec ${ }^{\mathrm{a}}$ \\ aProgram in Neuroscience and Department of Psychological and Brain Sciences, Indiana \\ University, Bloomington, IN 47405 \\ bDepartment of Pharmacology and Toxicology and Life Span Institute, University of Kansas, \\ Lawrence, KS 66045 \\ 'Department of Experimental Therapy, Franz-Penzoldt-Center, Friedrich-Alexander-University \\ Erlangen-Nürnbuerg, Germany \\ ${ }^{\mathrm{d} D e p a r t m e n t}$ of Medical Genetics, University of Tubingen, Tubingen, Germany \\ eDepartment of Chemistry, University of Kansas, Lawrence, KS 66045
}

\begin{abstract}
Altered neuronal activity in the striatum appears to be a key component of Huntington's disease (HD), a fatal, neurodegenerative condition. To assess this hypothesis in freely behaving transgenic rats that model HD (tgHDs), we used chronically implanted micro-wires to record the spontaneous activity of striatal neurons. We found that relative to wild-type controls, HD rats suffer from population-level deficits in striatal activity characterized by a loss of correlated firing and fewer episodes of coincident spike bursting between simultaneously recorded neuronal pairs. These results are in line with our previous report of marked alterations in the pattern of striatal firing in mouse models of HD that vary in background strain, genetic construct, and symptom severity. Thus, loss of coordinated spike activity in striatum appears to be a common feature of HD pathophysiology, regardless of HD model variability.
\end{abstract}

\section{Keywords}

Huntington's disease; Transgenic; Rat; Striatum; Medium spiny neurons; Electrophysiology; Crosscorrelation; Burst; Basal ganglia

\footnotetext{
(c) 2009 Elsevier Inc. All rights reserved.

Correspondence: G.V. Rebec, Program in Neuroscience, 1101 East $10^{\text {th }}$ St., Bloomington, IN 47405 (rebec@ indiana.edu, phone: 1-812-855-4832, fax: 1-812-4520.

Publisher's Disclaimer: This is a PDF file of an unedited manuscript that has been accepted for publication. As a service to our customers we are providing this early version of the manuscript. The manuscript will undergo copyediting, typesetting, and review of the resulting proof before it is published in its final citable form. Please note that during the production process errors may be discovered which could affect the content, and all legal disclaimers that apply to the journal pertain.
} 


\section{INTRODUCTION}

Huntington's disease (HD) is a dominantly inherited neurodegenerative disorder caused by a polyglutamine (CAG) expansion in the coding region of the huntingtin gene (HDCRG, 1993). Symptoms, including cognitive and emotional disturbances and adventitious movement, typically begin in middle age and progressively worsen until death (Harper, 1996). Although the hallmark of HD is neuronal death, emerging evidence suggests that disease progression results from a cascade of dysfunction at multiple levels of network organization that precede cell death (Cepeda et al., 2007; Palop et al., 2006). For example, several lines of transgenic HD mouse models show behavioral symptoms before cell loss (Levine et al., 2004). A key target of this cascade is GABAergic medium spiny neurons (MSNs) in striatum. In fact, progression of the HD phenotype is thought to be linked in part to changes in anatomical (Klapstein et al., 2001; Spires et al., 2004) and intrinsic properties (Cepeda et al., 2003; Joshi et al., 2009; Laforet et al., 2001; Milnerwood and Raymond, 2007) of MSNs that alter their capacity to processes information. However, one overlooked and underrepresented aspect of MSN dysfunction in HD is how the pathological cascade alters striatal neuronal output, which is a dynamic process critical for proper information throughput in basal ganglia circuits.

To help bridge this gap, we recently undertook the first-ever comprehensive assessment of how HD alters striatal communication in behaving HD models. We found that MSNs from both the R6/2 and knock-in (KI) mouse models, which differ in background strain, genetic construct, and symptom severity (Heng et al., 2008), have drastically altered spontaneous firing patterns compared to WTs (Miller et al., 2008). Because mouse models recapitulate multiple but varying aspects of human HD (Heng et al., 2008; Vonsattel, 2008), it is important to extend these findings to other relevant $\mathrm{HD}$ models.

Here we used a transgenic rat model of HD (tgHD) that carries a truncated huntingtin cDNA fragment with $51 \mathrm{CAG}$ repeats under the control of the endogenous promoter (von Hörsten et al., 2003). TgHD rats exhibit an adult-onset neuropathological phenotype; however, behavioral abnormalities, including a progressive cognitive, emotional, and motor symptomatology, precede this event (Cao et al., 2006; Nguyen et al., 2006; von Hörsten et al., 2003).

Histopatholocial alterations include huntingtin aggregates in nuclei, cytoplasm, dendrites and spines, axons, synaptic terminals, and mitochondria (Petrasch-Parwez et al., 2007). TgHD rats, moreover, show selective striatal neuron loss beginning at about 1.5 years in female (Kantor et al., 2006) and 1 year in male (Bode et al., 2008) tgHD rats. Thus, tgHDs closely resemble the neuropathological charcteristics of human HD. We recorded from MSNs of tgHD and WT rats as they behaved spontaneously in an open-field. Although no overt behavioral differences were distinguishable between genotypes, marked alterations in coordinated firing patterns emerged between the groups. Our results corroborate our previous findings from R6/2 and KI mice and suggest that dysregulated MSN firing patterns are a cardinal feature of HD, regardless of the genetic construct, phenotype, species, and extent of striatal pathology of the model.

\section{METHODS}

\section{Animals and Care}

Rats were sent to Indiana University from the University of Kansas, where a colony had previously been established. Male homozygous tgHD rats and wild-type (WT) control rats were used. The tgHD rats have a Sprague Dawley background and express a truncated huntingtin cDNA fragment with $51 \mathrm{CAG}$ (polyglutamine) repeats under control of the native rat huntingtin promoter. Genotyping was performed at Erlangen University using tail samples sent from the University of Kansas. On arrival at Indiana University, rats were housed individually in the departmental animal colony under standard conditions (12-h light/dark cycle with lights on at 06:00) with unrestricted access to food and water. Rats were allowed 1 wk of 
habitation before experimental procedures ensued. Five WT and four tgHD rats ranging in age between 10 and 15 months with mean age of $12.0 \pm 0.84$ and $13.25 \pm 0.75$ months $(P>0.05)$, respectively, were used. Both the housing and experimental use of animals followed the National Institutes of Health guidelines and were approved by the Institutional Animal Care and Use Committee.

\section{Surgical procedures}

Rats were anesthetized with Ketamine $(80 \mathrm{mg} / \mathrm{kg})$ and Xylazine $(10 \mathrm{mg} / \mathrm{kg})$, and mounted in a stereotaxic frame. The scalp was shaved, swabbed with betadine and $70 \%$ ethanol, and after lidocaine $(20 \mathrm{mg} / \mathrm{ml})$ was injected subcutaneously, an incision was made at the midline to expose the skull. A unilateral hole was drilled through the skull, the exposed dura was removed, and multi-wire electrodes (see below for details of electrodes) were lowered into either left or right dorsal striatum. Coordinates were $+1.0 \mathrm{~mm}$ anterior and $+2.5 \mathrm{~mm}$ lateral to bregma, and $4.5 \mathrm{~mm}$ ventral to the skull surface (Paxinos and Watson, 1998). The electrode assembly was secured on the skull with dental acrylic and bound to three anchor screws. Rats were allowed $1 \mathrm{wk}$ of post-surgical recovery, during which they were monitored for signs of pain and other health complications.

\section{Electrophysiology}

Electrode assemblies were made in-house and consisted of 16, $50 \mu \mathrm{m}$ Formvar-insulated stainless steel recording wires (California Fine Wire Company, Grover Beach, CA) and two, $50 \mu \mathrm{m}$ uninsulated stainless steel ground wires assembled in a custom fabricated hub. Electrode impedance was consistently $\sim 1 \mathrm{M} \Omega$. The electrode assembly was small, light-weight ( $2.9 \mathrm{~g})$, and well-tolerated by the rats so that they could behave freely.

All experiments were conducted during the light phase of the diurnal cycle and were $1 \mathrm{hr}$ in duration. During recording, the electrode assembly was connected to a light-weight flexible wire harness equipped with field-effect transistors that provide unity-gain current amplification for each of the micro-wires. Rats were placed in a force-plate actometer (see below for details). The force-plate was located inside a sound-attenuating and shielded recording chamber $(61 \mathrm{~L}$ $\times 51 \mathrm{~W} \times 71 \mathrm{H} \mathrm{cm}$ ). The harness was connected to a swiveling commutator, allowing the rats to behave freely in the actometer.

Extracellular spike activity was routed through preamplifiers with $500 \mathrm{X}$ gain, $154 \mathrm{~Hz}$ to 8.8 $\mathrm{kHz}$ band-pass filters, and digitized at $40 \mathrm{kHz}$ (Plexon, Dallas, TX). This system and its companion software Sort Client (Plexon) provide control over amplification, filtering, signal detection, spike-waveform discrimination, and storage. Units were sorted online prior to the recording session. Voltage thresholds $\geq 2.5$ times background noise were established, and waveform samples were collected to define a waveform template using principal component analysis. The raw signal for each unit was routed to an oscilloscope and audio monitor to facilitate unit discrimination by matching the analogue signal with the digitized template. To maximize the probability that units consisted of only one signal, autocorrelation and interspike interval (ISI) analyses for each unit was used to detect the presence of the absolute refractory period. Spike 2 software (Cambridge Electronic Design (Cambridge, England) was used to ensure unit isolations offline.

\section{Force-plate actometer}

The force-plate actometer is a computer-based instrument with high temporal and spatial resolution and has been used extensively to measure open-field behaviors of rats and mice (Fowler et al., 2001, 2007, 2009). For extensive details about the force-plate and methods for processing the data please refer to Fowler et al., 2001. Briefly, the force-plate consists of a low mass and highly stiff sensing surface plate measuring $42 \mathrm{~cm}$ on each side. Force samples from 
each of the four force transducers that supported the load plate were taken at a rate of 100 samples/s via a LabMaster interface controlled by a computer running custom written Free Pascal software. Force measurement resolution was 0.33 gram equivalent weights, and temporal resolution was the force sampling rate.

\section{Force-plate-derived behavioral analysis}

We used the force-plate actomoter, which also served as an open-field arena, to measure two very distinct behavioral attributes: low mobility bouts (LMBs) and ambulation. LMBs were defined as remaining in a virtual circle of $20.0 \mathrm{~mm}$ radius for $10.0 \mathrm{~s}$ or longer. Ambulatory activity, which encompasses all behavior not defined as a LMB (e.g., locomotion, rearing) was also quantified. Force-plate-derived measures of LMB and ambulation provide non-biased and quantifiable data to investigate overall spontaneous open-field behavior.

\section{Histology}

To verify electrode placement in dorsolateral striatum, rats received Ketamine and Xylazine ( $2 \mathrm{X}$ surgical dose) and a current pulse ( $30 \mu \mathrm{A}$ for $5 \mathrm{~s}$ ) was passed through each micro-wire to mark recording sites. Rats were transcardially perfused with saline followed by $10 \%$ potassium ferrocyanide $\left[\mathrm{K}_{4} \mathrm{Fe}(\mathrm{CN})_{6}\right]$ in $10 \%$ formalin to produce blue deposits at the site of the recording electrode ("Prussian blue" reaction). Brains were removed and cryoprotected in $30 \%$ sucrose dissolved in $10 \%$ formalin. The brains were then frozen, and coronal sections (50 $\mu \mathrm{M}$ ) were cut on a sliding microtome and mounted on gelatin subbed slides. The sections were stained with Cresyl violet and examined under a light microscope to confirm micro-wire location. Only recordings with clear electrode placements in the recording target area (Paxinos and Watson, 1998) were used for analysis.

\section{Data analysis}

Time stamps from neuronal data were analyzed by Neuroexplorer (Nex Technologies, Littleton, MA) and custom written Matlab scripts (Mathworks). All analyses were performed on single-unit data collected over the entire $1 \mathrm{hr}$ recording session. Firing rate was calculated by dividing the spike trains into $1 \mathrm{~s}$ bins (spikes/s). To assess spike-train variability, the coefficient of variation of interspike intervals (CV ISI) was calculated by dividing the standard deviation of all ISIs in a train by the mean ISI of the train. We used the Poisson surprise algorithm to quantify burst activity (Legenday and Salcman, 1985). Here we used a minimum burst surprise value of 5, which estimates that bursts occur $~ 150$ times $(\mathrm{P}<0.007)$ more frequently than would be expected in a Poisson spike train with the same mean firing rate. The surprise value provides an estimate of the statistical significance of each burst in the spike train. Therefore, the surprise value is an index of how intense or "surprising" the ISIs of a particular burst is compared with other ISIs in the same train. This method is a rigorous detector of bursts because it is not sensitive to fluctuations in average firing rate and treats each spike train as an independent entity. The method, moreover, has been well established for detecting bursts in striatum and other basal-ganglia structures (Aldridge and Gilman, 1991; Miller et al., 2008; Wichmann and Soares, 2006).

Coincident bursts were defined as bursts from two units that overlap in time (Lisman, 1997, Miller et al., 2008). The mean time that bursts were coincident (coincidence duration), was also calculated. Coincident bursting and coincidence duration were determined for each pairwise comparison in each session.

To assess correlated firing between two spike trains, cross-correlation histograms (CCHs) were constructed for each pair-wise comparison (Perkel et al., 1967) in each $1 \mathrm{hr}$ recording session. All CCHs were constructed based on $1 \mathrm{~ms}$ bins and a $\pm 0.5 \mathrm{~s}$ time lag from the zero bin. The $\mathrm{CCHs}$ were smoothed using a Gaussian filter with a bin width of 3. Significant peaks, which 
indicate correlated firing, were identified using a 99\% confidence interval by assuming the null hypothesis that each spike train is a Poisson process and that firing between neuronal pairs is independent (i.e., flat cross-correlogram) (Abeles, 1982).

\section{Statistical analysis}

GraphPad Prism (GraphPad Software, San Diego, CA) was used for all statistical analyses. Parametric Student's t-tests were used to compare age and behavior of rats. Mann-Whitney U tests were used to compare firing rate, CV ISI, and bursting parameters between tgHD and WT rats. Nonparametric statistics are appropriate because of the significant deviation from normality and a lack of homogeneous variances in spike-train samples (Zar, 2009). Therefore, our electrophysiological data represent the median of the mean values. Pearson's chi-square $\left(\chi^{2}\right)$ test was used to determine differences in the proportions of coincident bursting and crosscorrelations with significant peaks between unit pairs in tgHD and WT rats. The alpha level of significance was $P<0.05$.

\section{RESULTS}

\section{Force-plate-derived behavioral analysis}

Our goal was to characterize overall firing patterns while rats engaged in spontaneous openfield behaviors. We used a force-plate actometer to measure two distinct behavioral events. Low mobility bouts (LMBs; each defined as remaining in a virtual circle of $20.0 \mathrm{~mm}$ radius for $10.0 \mathrm{~s}$ or longer) predominated the sessions and represent periods of low activity.

Ambulatory activity, which encompassed all behaviors not defined as a LMB (e.g., locomotion, rearing) and represents periods of behavioral activation, was also quantified. Force-platederivation of LMBs and ambulation provide non-biased and quantifiable data to investigate overall spontaneous open-field behavior. The percent time engaged in these measures was not different between the genotypes (Fig. 1; $P>0.05$ ). Therefore, our electrophysiological data are not likely compromised by overt differences in open-field behaviors between WT and tgHD rats.

\section{Single-unit isolations represent putative MSNs}

We recorded 34 and 38 individually isolated single-units from WT and tgHD rats, respectively. Histological analysis confirmed that micro-wire electrode placements were in dorsal striatum (Fig. 2a). Waveform amplitudes ranged from $50-100 \mu \mathrm{V}$ and durations ranged from $0.5-0.8$ $\mathrm{ms}$. In addition, waveforms were asymmetric; the duration of the late positive phase of all waveforms exceeded that of the early negative deflection (Fig. 2b). Analysis of peak and valley widths indicated a common waveform shape in all cells. Although firing rate was somewhat variable (see below), all neurons exhibited burst firing interrupted by periods of low firing or near-quiescent activity. Indicative of burst activity, inter-spike interval (ISI) histograms showed unimodal and positively skewed profiles (Fig. 2c). These waveform and spike-pattern characteristics have been used extensively to classify extracellularly recorded MSNs (Berke et al., 2004,2008;Costa et al., 2004;Courtemanche et al., 2003;Miller et al., 2008;Sharrot et al., 2009). Thus, our units likely represent a homogenous population of striatal MSNs.

\section{Firing pattern of individual MSNs}

Our first analysis focused on spontaneous firing patterns of individual MSNs. A significant increase in rate relative to tgHD rats emerged in WTs (Fig. 3a; $P<0.05$ ). Consistent with this finding, ISIs were shorter in WTs compared to tgHDs (Table $1 ; P<0.05$ ). However, spiketrain variability, which is measured by CV ISI, was not different between the genotypes (Table 1). Thus, although WTs had higher firing rates, the pattern of activity was equally variable compared to tgHDs. Consistent with comparable CV ISIs, we found no differences in any other 
spike pattern metric, including bursts/min, percentage of spikes included in bursts, burst duration, inter-burst interval, ISI in bursts, and burst surprise (Table 1). It appears, therefore, that overall spontaneous firing patterns, other than rate, in individual MSNs are similar in WT and tgHD rats.

\section{Coincident bursting between MSN pairs}

We next aimed to investigate the temporal relationship of bursts among local populations of MSN unit pairs (i.e., all pair-wise comparisons for each animal). We previously found a reduction in coincident bursting, which measures bursts that overlap in time between unit pairs, in MSNs of R6/2 and KI mice compared to their respective WTs (Miller et al., 2008). Here, we recorded a large number of unit pairs: 127 in WT and 137 in tgHD rats. Intriguingly, we found a marked reduction in the number of coincident bursts in tgHD compared to WTs (Fig. $3 \mathrm{~b} ; P<0.001)$. A separate $\chi^{2}$ analysis revealed that the distribution of coincident bursting between unit pairs was also different in WT compared to tgHDs $\left(\chi^{2}=33.1, P<0.001\right)$. For example, $99.2 \%$ of unit pairs in WT had at least one instance of coincident bursting between each pair in the recording session. In contrast, only $75.2 \%$ of unit pairs in tgHDs exhibited coincident bursts. Coincidence duration, which is defined as the mean time that bursts were coincident, was not different $(P>0.05)$ between WT $\left(0.30 \mathrm{~s}, 0.05-1.2\right.$; median, $25^{\text {th }}-75^{\text {th }}$ percentile) and tgHDs $(0.27 \mathrm{~s}, 0.02-3.93)$.

Figure 4 shows representative examples of simultaneously recorded spike rasters recorded from WT and tgHD rats. Note that WT spike trains exhibit somewhat faster firing rates compared to tgHD trains (see Fig. 3a). Bursts are indicated by solid lines above each train. Coincident bursting among many unit pairs is a clear feature of WT trains; a phenomenon that is nearly absent in tgHDs.

\section{Correlated firing in MSN pairs}

Temporally coherent spike activity between MSNs is thought to be important for striatal information processing and is likely driven in part by synchronized inputs from corticostriatal fibers (Sharott et al., 2009). Interestingly, loss of correlated MSN activity is prominent in R6/2 and KI mouse models of HD (Miller et al., 2008) and may be attributable to dysfunction in the corticostriatal pathway (Cepeda et al., 2007). Therefore, to investigate the temporal relationship of spikes recorded among local populations of MSN unit pairs, we constructed crosscorrelation histogram $(\mathrm{CCH})$ matrices for all pair-wise comparisons (same number of unit pairs as above). Peaks that exceed the upper $99 \%$ confidence limit in the $\mathrm{CCH}$ indicate correlated firing between pairs of units, whereas peaks below this limit are considered non-correlated (Abeles, 1982). We found that a large proportion of unit pairs showed correlated firing in WT rats $(52.0 \%)$ and this was drastically reduced in tgHDs $(19.7 \%)\left(\chi^{2}=30.1, P<0.001\right)$. The correlated activity, moreover, was not oscillatory; rather, the peaks were bimodal with asymptotes near the zero bin. Representative $\mathrm{CCH}$ matrices for simultaneously recorded units from WT and tgHDs, respectively, are shown in Fig. 5.

\section{DISCUSSION}

Our results indicate a marked disruption in coordinated firing patterns in dorsal striatum of behaving tgHD compared to WT rats. This investigation took advantage of tgHD rats as they recapitulate several important features of human HD (for review see Heng et al., 2008). For example, tgHD rats exhibit an adult-onset HD phenotype, including slowly progressive impairments in motor, cognitive, and affective behavior, nuclear inclusions, neuropil aggregates, striatal atrophy, enlarged ventricles, and reduced striatal volume (Kantor et al., 2006; Nguuyen et al., 2006; von Hörsten et al., 2003). Interestingly, however, behavioral abnormalities precede polyQ recruitment sites and striatal atrophy (Nguyen et al., 2006), which 
is consistent with other models of the disease (Carter et al., 1999; Menalled et al., 2003; Slow et al., 2003). We used rats at approximately middle age, which is a time that many of the key HD phenotypes and pathologies develop in tgHDs (von Hörsten et al., 2003) and in the human disease (Vonsattel and DiFiglia, 1998).

Although younger tgHD rats show no resting tremor, ataxia, clasping, dyskinesia, or seizures (von Hörsten et al., 2003), at a time near middle age they begin to show discrete impairments in performance on motor-skill tasks that measure fine motor coordination, such as rotarod (6 - 10 months) and beam walking (9 months) (Nguyen et al., 2006; von Hörsten et al., 2003). To circumvent differences in behavior during motor-skill tasks, we recorded rats in an open-field. The goal of our behavioral analysis was to ensure that MSN recordings reflected patterns of neuronal firing during spontaneous gross motor activity (e.g., locomotion) and was not compromised by differences in overt motor output between the genotypes. Force-plate-derived analysis of open-field behavior revealed no differences in behavioral activation in tgHDs compared to WTs (Fig. 1). Generally, the rats engaged in quiet-like rest interspersed with subtle movements, including head movements and slight postural changes. These periods were considered LMBs and defined as remaining in a virtual circle of $20.0 \mathrm{~mm}$ radius for $10.0 \mathrm{~s}$ or longer. Ambulation occurred less frequently but often interrupted periods of LMBs. Our openfield data are consistent with evidence that the motor phenotype of tgHDs is subtle and appears later in disease progression unless tested on specific measures of motor coordination. It appears, therefore, that differences in unit data are not a result of overall gross differences in behavioral activation during recording sessions.

Extracellular recordings rely on waveform and spike pattern characteristics to determine the putative cellular origin of units. Several criteria suggest that our neuronal sample represents MSNs. First, MSNs are the most common neuron in striatum, accounting for $>90 \%$ of the neuronal population (Bolam et al., 2000). Second, MSNs display asymmetric waveforms, in which the duration of the late positive phase exceeds the early negative deflection (see Fig. 2B; Berke et al., 2004; Sharott et al., 2009). Lastly, MSN firing patterns exhibit bursting interspersed with periods of low frequency or near quiescence (see Fig. 4; Wilson and Groves, 1981). These waveform- and spike-pattern profiles characterize our neuronal sample. In contrast, tonically active neurons, which are the presumed cholinergic interneurons comprising only 5\% of the neuronal population in striatum (Graveland and DiFiglia, 1985), have high firing frequencies, lack burst activity, and exhibit long duration and high amplitude waveforms (Courtemanche et al., 2003; Sharrot et al., 2009; Wilson et al., 1990). We also discount the possibility that parvalbumin-staining GABAergic interneurons contributed to our sample, as they have high firing frequencies and short duration waveforms (Berke et al., 2004; Sharrot et al., 2009). Based on these criteria, we classified our neuronal sample as putative MSNs.

Excluding rate, we found no differences in the firing patterns of individual MSNs between tgHD and WTs (see table 1). We previously reported increased firing rate, and decreased burst rate, percentage of spikes in bursts, and burst surprise in the R6/2 mouse model of HD relative to WT (Miller et. al., 2008;Rebec et al., 2006). However, in the KI model, which is a more subtle HD model (i.e., less rapidly progressing phenotype) than R6/2s (Carter et al., 1999; Menalled et al., 2003), the only differences that emerged were the percentage of spikes in bursts and burst surprise (Miller et al., 2008). It is likely that there were no differences in individual MSN firing patterns in our tgHD rats because, like the less severe HD progression exhibited by KIs, they have a late-onset and more slowly progressive behavioral and pathological phenotype than R6/2 mice. Similarly, recordings from pre-frontal cortex in R6/2 mice has revealed marked differences in firing patterns of individual neurons that were completely absent in KIs (Walker et al., 2008). Thus, it appears that the more subtle phenotype of tgHDs correlates to a less severe striatal deficit in neuronal processing at the level of individual MSNs. 
Higher firing rates in WT compared to tgHDs is intriguing. One explanation is an age effect. For example, striatal neurons in aged ( 24 months of age) F344 rats exhibit significantly higher firing rates than rats at $\sim 6$ months of age (Stanford and Gerhardt, 2004). Indeed, firing rates in our WTs were somewhat higher than what is typically reported (see Berke et al., 2004; Sharott et al., 2009). This, however, does not explain the difference between genotype. Alternatively, our neuronal samples in WT compared to tgHD could reflect different MSN populations, although it is unclear if MSNs are differentially affected in rodent models of HD.

Despite similar firing patterns at the single-cell level, we found that coincident bursting was significantly decreased in tgHD compared to WTs. Thus, it is not that tgHD MSNs loose the capacity to generate burst activity, rather, coordinated bursting between unit pairs in local striatal populations is altered. No difference in coincidence duration between genotypes (i.e., the duration of the overlap) supports this view. For example, when a coincident burst occurs in either of the genotypes, there is no difference in the burst structure (e.g. burst duration, ISI in bursts) of the unit pairs (see Table 1). Coincident bursts are thought to optimize neuronal signaling between neurons by increasing the signal-to-noise ratio and avoiding synaptic transmission failure (Lisman, 1997). It is conceivable that loss of coincident bursting in tgHDs, and other HD models (Miller et al., 2008), deteriorates the quality of information transmission between striatum and its targets.

Pair-wise analyses of the temporal relationship of individual spikes between MSN pairs revealed that correlated firing is a prominent feature of WT MSNs that is reduced in tgHDs. Similar to coincident bursting, correlated firing is thought to enhance the flow of neuronal information (Abeles et al., 1994; Salinas and Sejnowski, 2001). Significant cross-correlations between MSNs do not necessarily reflect a monosynaptic connection (Nowak and Bullier, 2000). Indeed, evidence suggests that correlated MSN activity is produced by synchronized locking to excitatory input from populations of cortical neurons (Sharrot et al., 2009). This explains the fact that pairs of inhibitory MSNs in our sample exhibited only positively correlated peaks near the zero lag in the $\mathrm{CCH}$ (i.e., no significant negative correlations occurred). Therefore, we suggest that altered corticostriatal communication, which is a major problem in many HD models (Cepeda et al., 2007), may contribute to reduced correlated activity between MSNs in tgHDs, as well as MSNs in R6/2 and KIs (Miller et al., 2008). In light of this concept, we have previously shown reduced correlated firing in pre-frontal cortex of R6/2 and KI mice (Walker et al., 2008). Because striatal neurons require temporally convergent cortical excitation to bring them into a depolarized up-state to elicit firing (Wilson and Kawaguchi, 1996), it is conceivable that loss of correlated activity in cortex could in turn result in loss of correlated activity in striatum.

Overall, our results support our previous findings and suggest that loss of coordinated firing patterns during spontaneous behaviors is a cardinal feature of HD pathophysiology. In fact, these findings transcend differences in genetic construct, phenotype, species, and degree of striatal pathology of the HD model. It will be important to identify the main factors that contribute to altered neuronal firing patterns in the corticostriatal pathway so that promising therapeutic strategies can be developed.

\section{Acknowledgments}

We thank Faye Caylor for editorial and administrative support, Paul Langley and Jason Ummel for technical assistance, and Dr. Youssef Sari for the photomicrograph. This work was supported by National Institute of Neurological Disorders and Stroke Grants R01 NS-35663 (G.V.R.) and F31 NS-064791 (B.R.M.) and by a grant from the Hereditary Disease Foundation (M.A.J). 


\section{REFERENCES}

Abeles M. Quantification, smoothing, and confidence limits for single-units' histograms. J. Neurosci. Methods 1982;5:317-25. [PubMed: 6285087]

Aldridge JW, Gilman S. The temporal structure of spike trains in the primate basal ganglia: afferent regulation of bursting demonstrated with precentral cerebral cortical ablation. Brain Res 1991;543:123-38. [PubMed: 2054667]

Berke JD. Uncoordinated firing rate changes of striatal fast-spiking interneurons during behavioral task performance. J. Neurosci 2008;28:10075-80. [PubMed: 18829965]

Berke JD, Okatan M, Skurski J, Eichenbaum HB. Oscillatory entrainment of striatal neurons in freely moving rats. Neuron 2004;43:883-96. [PubMed: 15363398]

Bode FJ, Stephan M, Suhling H, Pabst R, Straub RH, Raber KA, Bonin M, Nguyen HP, Riess O, Bauer A, Sjoberg C, Petersen A, von Horsten S. Sex differences in a transgenic rat model of Huntington's disease: decreased 17beta-estradiol levels correlate with reduced numbers of DARPP32+ neurons in males. Hum. Mol. Genet 2008;17:2595-609. [PubMed: 18502785]

Bolam JP, Hanley JJ, Booth PA, Bevan MD. Synaptic organisation of the basal ganglia. J. Anat 2000;196 (Pt 4):527-42. [PubMed: 10923985]

Cao C, Temel Y, Blokland A, Ozen H, Steinbusch HW, Vlamings R, Nguyen HP, von Horsten S, Schmitz $\mathrm{C}$, Visser-Vandewalle V. Progressive deterioration of reaction time performance and choreiform symptoms in a new Huntington's disease transgenic ratmodel. Behav. Brain. Res 2006;170:257-61. [PubMed: 16569446]

Carter RJ, Lione LA, Humby T, Mangiarini L, Mahal A, Bates GP, Dunnett SB, Morton AJ. Characterization of progressive motor deficits in mice transgenic for the human Huntington's disease mutation. J. Neurosci 1999;19:3248-57. [PubMed: 10191337]

Cepeda C, Andre VM, Yamazaki I, Wu N, Kleiman-Weiner M, Levine MS. Differential electrophysiological properties of dopamine D1 and D2 receptor-containing striatal medium-sized spiny neurons. Eur. J. Neurosci 2008;27:671-82. [PubMed: 18279319]

Cepeda C, Hurst RS, Calvert CR, Hernandez-Echeagaray E, Nguyen OK, Jocoy E, Christian LJ, Ariano MA, Levine MS. Transient and progressive electrophysiological alterations in the corticostriatal pathway in a mouse model of Huntington's disease. J. Neurosci 2003;23:961-9. [PubMed: 12574425]

Cepeda C, Wu N, Andre VM, Cummings DM, Levine MS. The corticostriatal pathway in Huntington's disease. Prog. Neurobiol 2007;81:253-71. [PubMed: 17169479]

Costa RM, Cohen D, Nicolelis MA. Differential corticostriatal plasticity during fast and slow motor skill learning in mice. Curr. Biol 2004;14:1124-34. [PubMed: 15242609]

Courtemanche R, Fujii N, Graybiel AM. Synchronous, focally modulated beta-band oscillations characterize local field potential activity in the striatum of awake behaving monkeys. J. Neurosci 2003;23:11741-52. [PubMed: 14684876]

Deng YP, Albin RL, Penney JB, Young AB, Anderson KD, Reiner A. Differential loss of striatal projection systems in Huntington's disease: a quantitative immunohistochemical study. J Chem Neuroanat 2004;27:143-64. [PubMed: 15183201]

Fowler SC, Birkestrand BR, Chen R, Moss SJ, Vorontsova E, Wang G, Zarcone TJ. A force-plate actometer for quantitating rodent behaviors: illustrative data on locomotion, rotation, spatial patterning, stereotypies, and tremor. J. Neurosci. Methods 2001;107:107-24. [PubMed: 11389948]

Fowler SC, Covington HE 3rd, Miczek KA. Stereotyped and complex motor routines expressed during cocaine self-administration: results from a 24-h binge of unlimited cocaine access in rats. Psychopharmacology (Berl) 2007;192:465-78. [PubMed: 17333134]

Fowler SC, Miller BR, Gaither TW, Johnson MA, Rebec GV. Force-plate quantification of progressive behavioral deficits in the R6/2 mouse model of Huntington's disease. Behav Brain Res 2009;202:130-7. [PubMed: 19447289]

Graveland GA, DiFiglia M. The frequency and distribution of medium-sized neurons with indented nuclei in the primate and rodent neostriatum. Brain. Res 1985;327:307-11. [PubMed: 3986508]

Harper, PS. Huntington's disease. W.B. Saunders; London: 1991.

Heng MY, Detloff PJ, Albin RL. Rodent genetic models of Huntington disease. Neurobiol. Dis 2008;32:1-9. [PubMed: 18638556] 
Huntington's Disease Collaborative Research Group. A novel gene containing a trinucleotide repeat that is expanded and unstable on Huntington's disease chromosomes. Cell 1993;72:971-983. [PubMed: 8458085]

Joshi PR, Wu NP, Andre VM, Cummings DM, Cepeda C, Joyce JA, Carroll JB, Leavitt BR, Hayden MR, Levine MS, Bamford NS. Age-dependent alterations of corticostriatal activity in the YAC128 mouse model of Huntington disease. J. Neurosci 2009;29:2414-27. [PubMed: 19244517]

Kantor O, Temel Y, Holzmann C, Raber K, Nguyen HP, Cao C, Turkoglu HO, Rutten BP, VisserVandewalle V, Steinbusch HW, Blokland A, Korr H, Riess O, von Horsten S, Schmitz C. Selective striatal neuron loss and alterations in behavior correlate with impaired striatal function in Huntington's disease transgenic rats. Neurobiol. Dis 2006;22:538-47. [PubMed: 16480885]

Klapstein GJ, Fisher RS, Zanjani H, Cepeda C, Jokel ES, Chesselet MF, Levine MS. Electrophysiological and morphological changes in striatal spiny neurons in R6/2 Huntington's disease transgenic mice. J. Neurophysiol 2001;86:2667-77. [PubMed: 11731527]

Laforet GA, Sapp E, Chase K, McIntyre C, Boyce FM, Campbell M, Cadigan BA, Warzecki L, Tagle DA, Reddy PH, Cepeda C, Calvert CR, Jokel ES, Klapstein GJ, Ariano MA, Levine MS, DiFiglia $\mathrm{M}$, Aronin N. Changes in cortical and striatal neurons predict behavioral and electrophysiological abnormalities in a transgenic murine model of Huntington's disease. J. Neurosci 2001;21:9112-23. [PubMed: 11717344]

Legendy CR, Salcman M. Bursts and recurrences of bursts in the spike trains of spontaneously active striate cortex neurons. J. Neurophysiol 1985;53:926-39. [PubMed: 3998798]

Levine MS, Cepeda C, Hickey MA, Fleming SM, Chesselet MF. Genetic mouse models of Huntington's and Parkinson's diseases: illuminating but imperfect. Trends Neurosci 2004;27:691-7. [PubMed: 15474170]

Lisman JE. Bursts as a unit of neural information: making unreliable synapses reliable. Trends Neurosci 1997;20:38-43. [PubMed: 9004418]

Menalled LB, Sison JD, Dragatsis I, Zeitlin S, Chesselet MF. Time course of early motor and neuropathological anomalies in a knock-in mouse model of Huntington's disease with $140 \mathrm{CAG}$ repeats. J. Comp. Neurol 2003;465:11-26. [PubMed: 12926013]

Miller BR, Walker AG, Shah AS, Barton SJ, Rebec GV. Dysregulated information processing by medium spiny neurons in striatum of freely behaving mouse models of Huntington's disease. J. Neurophysiol 2008;100:2205-16. [PubMed: 18667541]

Milnerwood AJ, Raymond LA. Corticostriatal synaptic function in mouse models of Huntington's disease: early effects of huntingtin repeat length and protein load. J. Physiol 2007;585:817-31. [PubMed: 17947312]

Nguyen HP, Kobbe P, Rahne H, Worpel T, Jager B, Stephan M, Pabst R, Holzmann C, Riess O, Korr H, Kantor O, Petrasch-Parwez E, Wetzel R, Osmand A, von Horsten S. Behavioral abnormalities precede neuropathological markers in rats transgenic for Huntington's disease. Hum. Mol. Genet 2006;15:3177-94. [PubMed: 16984963]

Nowak, LG.; Bullier, J. Time in the brain. Harwood Academic; Amsterdam: 2000. Cross-correlograms for neuronal spike trains. Different types of temporal correlatino in the neocortex, their origin and significance; p. 53-96.

Palop JJ, Chin J, Mucke L. A network dysfunction perspective on neurodegenerative diseases. Nature 2006;443:768-73. [PubMed: 17051202]

Paxinos, G.; Watson, C. The rat brain in stereotaxic coordinates. Academic Press; New York: 1998.

Perkel DH, Gerstein GL, Moore GP. Neuronal spike trains and stochastic point processes. II. Simultaneous spike trains. Biophys. J 1967;7:419-40. [PubMed: 4292792]

Petrasch-Parwez E, Nguyen HP, Lobbecke-Schumacher M, Habbes HW, Wieczorek S, Riess O, Andres KH, Dermietzel R, Von Horsten S. Cellular and subcellular localization of Huntingtin [corrected] aggregates in the brain of a rat transgenic for Huntington disease. J. Comp. Neurol 2007;501:71630. [PubMed: 17299753]

Rebec GV, Conroy SK, Barton SJ. Hyperactive striatal neurons in symptomatic Huntington R6/2 mice: variations with behavioral state and repeated ascorbate treatment. Neuroscience 2006;137:327-36. [PubMed: 16257492] 
Salinas E, Sejnowski TJ. Correlated neuronal activity and the flow of neural information. Nat. Rev. Neurosci 2001;2:539-50. [PubMed: 11483997]

Sharott A, Moll CK, Engler G, Denker M, Grun S, Engel AK. Different subtypes of striatal neurons are selectively modulated by cortical oscillations. J. Neurosci 2009;29:4571-85. [PubMed: 19357282]

Slow EJ, van Raamsdonk J, Rogers D, Coleman SH, Graham RK, Deng Y, Oh R, Bissada N, Hossain SM, Yang YZ, Li XJ, Simpson EM, Gutekunst CA, Leavitt BR, Hayden MR. Selective striatal neuronal loss in a YAC128 mouse model of Huntington disease. Hum. Mol. Genet 2003;12:155567. [PubMed: 12812983]

Spires TL, Grote HE, Garry S, Cordery PM, Van Dellen A, Blakemore C, Hannan AJ. Dendritic spine pathology and deficits in experience-dependent dendritic plasticity in R6/1 Huntington's disease transgenic mice. Eur. J. Neurosci 2004;19:2799-807. [PubMed: 15147313]

Stanford JA, Gerhardt GA. Aged F344 rats exhibit altered electrophysiological activity in locomotorunrelated but not locomotor-related striatal neurons. Neurobiol. Aging 2004;25:509-15. [PubMed: 15013572]

von Horsten S, Schmitt I, Nguyen HP, Holzmann C, Schmidt T, Walther T, Bader M, Pabst R, Kobbe P, Krotova J, Stiller D, Kask A, Vaarmann A, Rathke-Hartlieb S, Schulz JB, Grasshoff U, Bauer I, Vieira-Saecker AM, Paul M, Jones L, Lindenberg KS, Landwehrmeyer B, Bauer A, Li XJ, Riess O. Transgenic rat model of Huntington's disease. Hum. Mol. Genet 2003;12:617-24. [PubMed: 12620967]

Vonsattel JP. Huntington disease models and human neuropathology: similarities and differences. Acta Neuropathol 2008;115:55-69. [PubMed: 17978822]

Vonsattel JP, DiFiglia M. Huntington disease. J. Neuropathol. Exp Neurol 1998;57:369-84. [PubMed: 9596408]

Walker AG, Miller BR, Fritsch JN, Barton SJ, Rebec GV. Altered information processing in the prefrontal cortex of Huntington's disease mouse models. J. Neurosci 2008;28:8973-82. [PubMed: 18768691]

Wichmann T, Soares J. Neuronal firing before and after burst discharges in the monkey basal ganglia is predictably patterned in the normal state and altered in parkinsonism. J. Neurophysiol 2006;95:212033. [PubMed: 16371459]

Wilson CJ, Chang HT, Kitai ST. Firing patterns and synaptic potentials of identified giant aspiny interneurons in the rat neostriatum. J. Neurosci 1990;10:508-19. [PubMed: 2303856]

Wilson CJ, Groves PM. Spontaneous firing patterns of identified spiny neurons in the rat neostriatum. Brain Res 1981;220:67-80. [PubMed: 6168334]

Wilson CJ, Kawaguchi Y. The origins of the two-state spontaneous membrane potential fluctuaitons of neostrital spiny neurons. J. Neurosci 1996;16:2397-2410. [PubMed: 8601819]

Zar, JH. Biostatistical analysis. Vol. Ed 5. Prentice Hall; Upper Saddle River, NJ: 2009. 


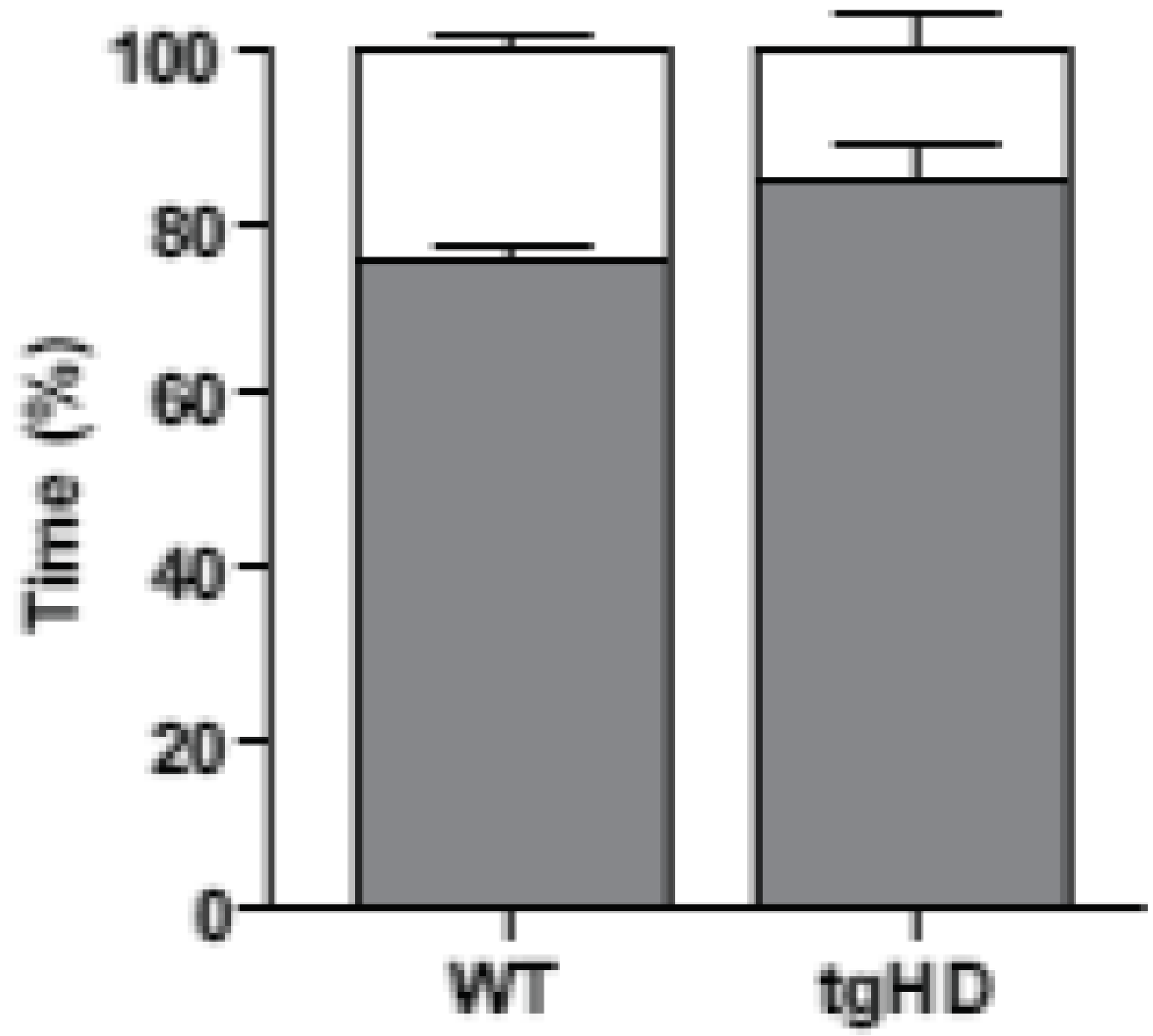

Fig. 1.

Force-plate derived behavioral analysis. Behavior was coded as percent time engaged in either LMB (each defined as remaining in a virtual circle of $20.0 \mathrm{~mm}$ radius for $10.0 \mathrm{~s}$ or longer) or ambulation, which encompasses all behaviors not defined as LMBs) (see results for details). There was no difference in LMBs or ambulation between the genotypes. (t-test, $P>0.05, \mathrm{n}=$ 5 WTs and $n=4$ tgHDs, mean \pm SEM). 

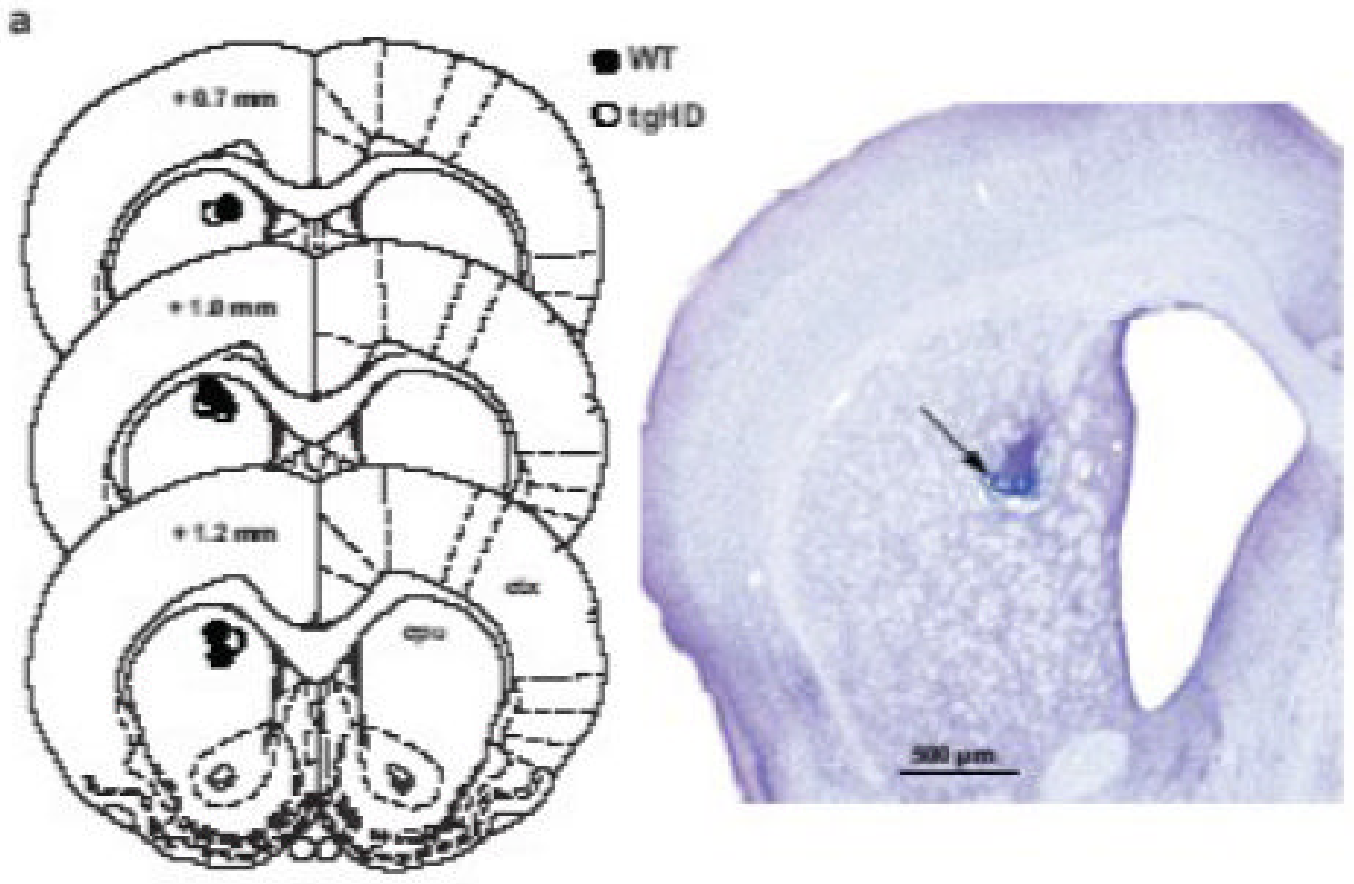

b

WT
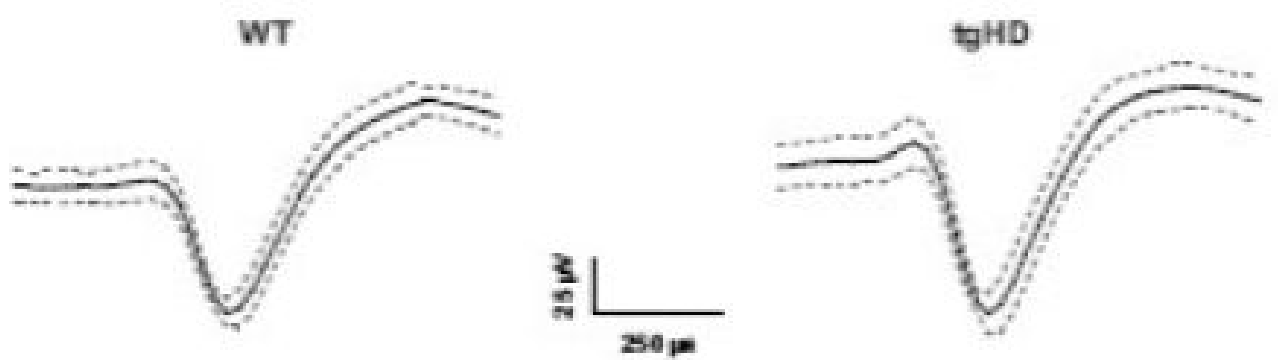

c
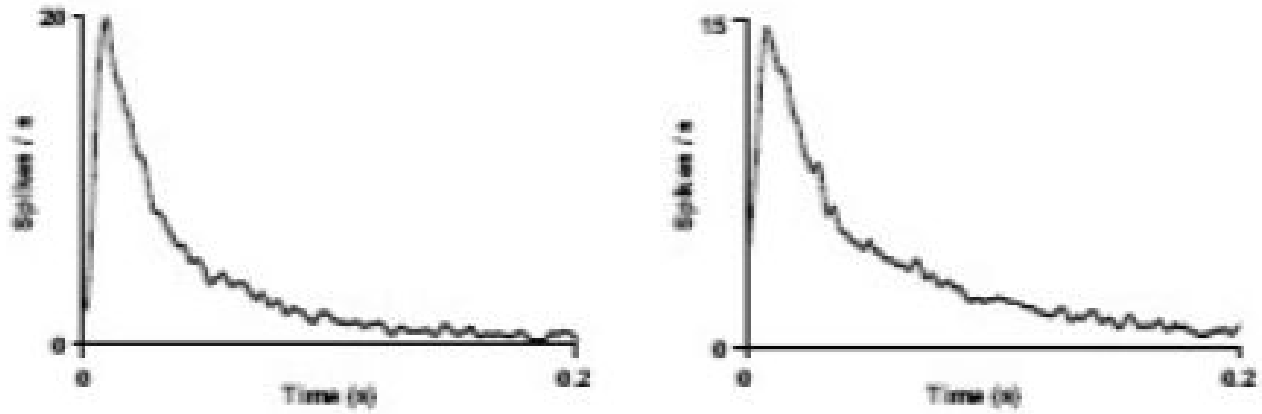

Fig. 2.

Classification of putative medium spiny neurons (MSNs) in striatum. (a, left) coronal diagrams of rat brain showing the location of histologically verified recording sites for each animal (modified from Paxinos and Watson, 1998). Numbers indicate distance (mm) anterior to bregma $(\mathrm{ctx}=$ cortex, $\mathrm{cpu}=$ striatum $)$. $(\mathrm{a}$, right $)$ photomicrograph of a representative coronal section showing the recording site (arrow) in striatum. (b) waveforms recorded from putative MSNs isolated in striatum of WT and tgHD rats. The solid black lines represent the mean of 50 consecutive waveforms and the dotted gray lines indicate $\pm 1 \mathrm{SD}$. Waveforms from both genotypes had comparable peak/valley widths and durations. (c) interspike interval (ISI) 
histograms (1 ms bins) corresponding to the units directly above in $b$. Note skewed profiles in both histograms indicate burst firing. 
a

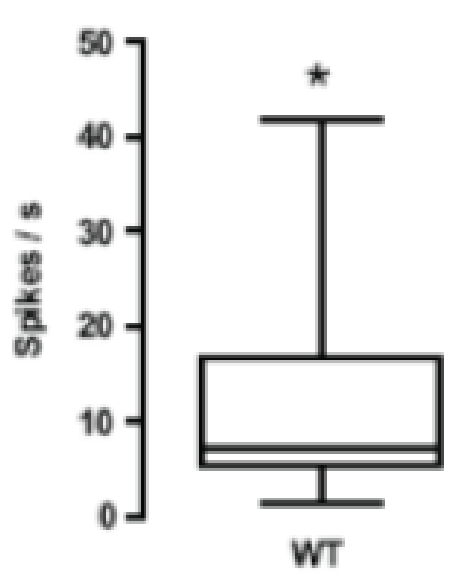

b

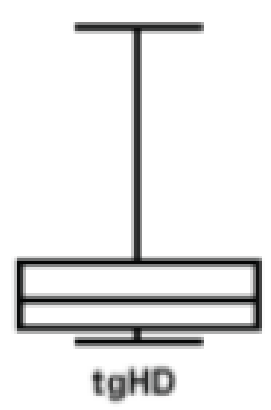

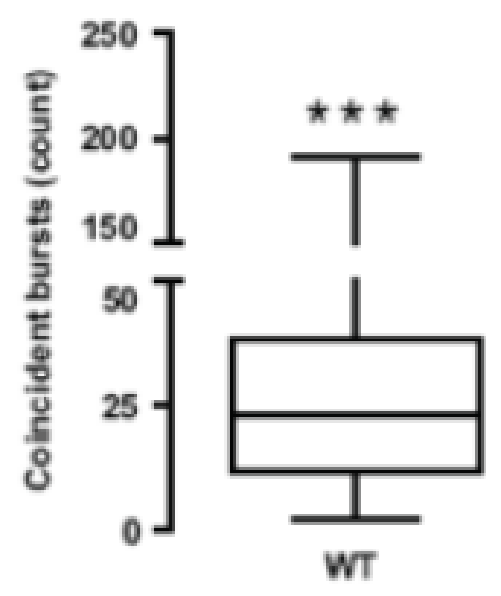

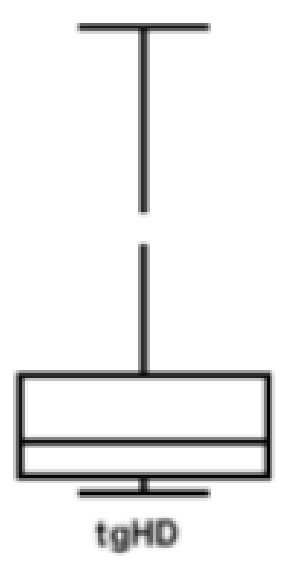

Fig. 3.

Firing pattern of MSNs. (a) Spike rate was higher in WT compared to tgHDs. (b) WT unit pairs, (i.e., all pair-wise comparisons) displayed more coincident bursts than tgHDs. Data are illustrated as box-and-whiskers plots. The box extends from the $25^{\text {th }}$ to the $75^{\text {th }}$ percentile with the line at the median $\left(50^{\text {th }}\right.$ percentile). The whiskers represent the minimum and maximum values. (Mann-Whitney U test, $* P<0.05, * * * P<0.001$ ). 


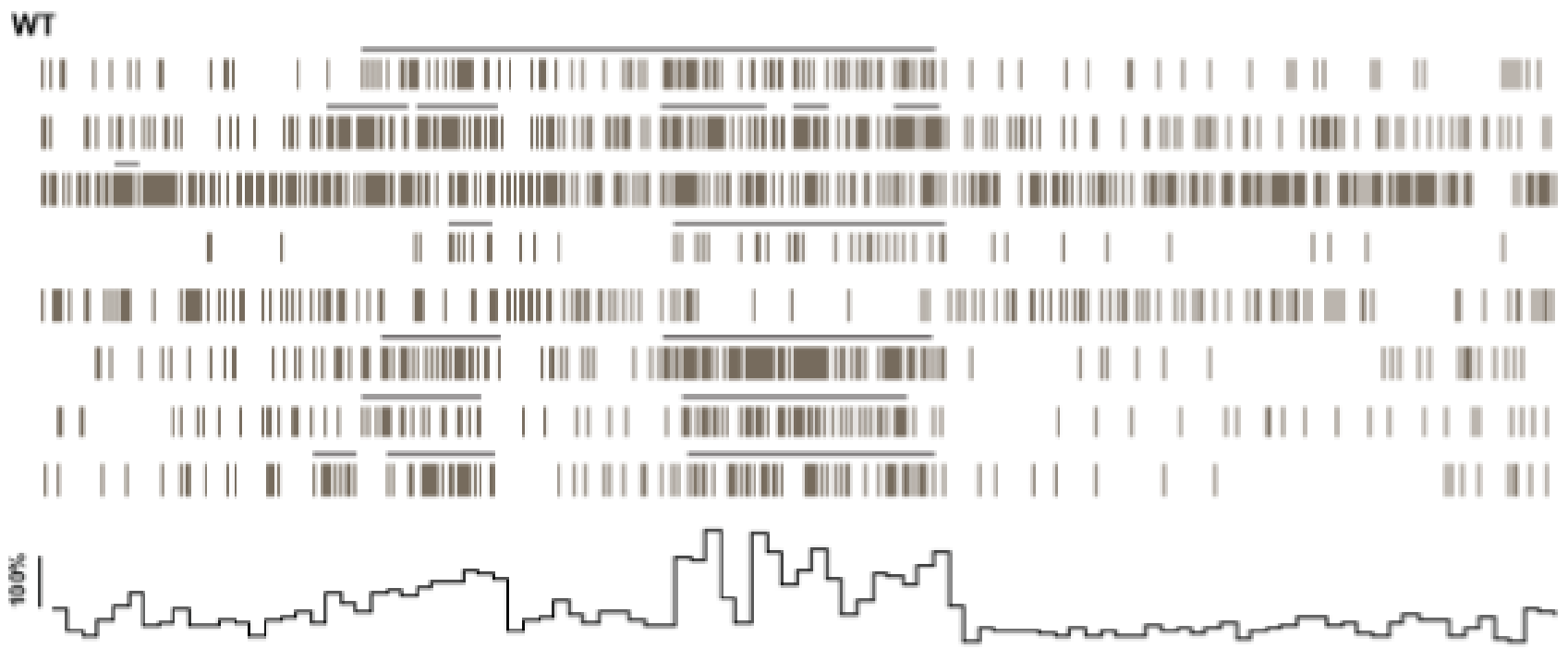

$\operatorname{tgHD}$

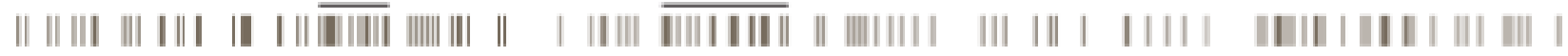

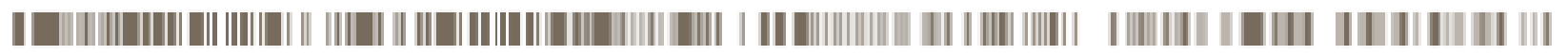

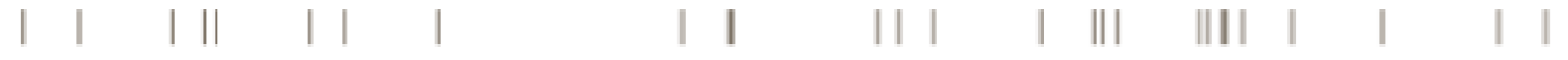

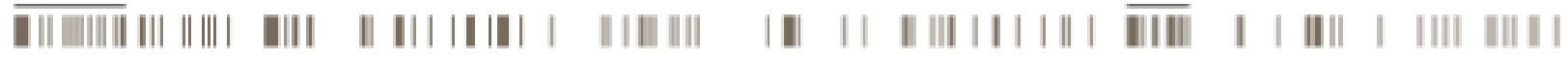

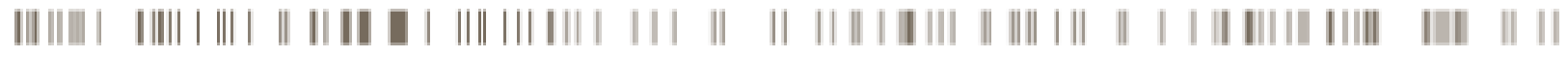

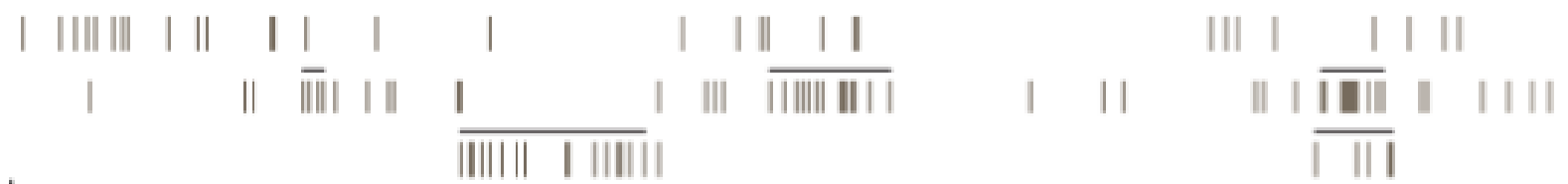

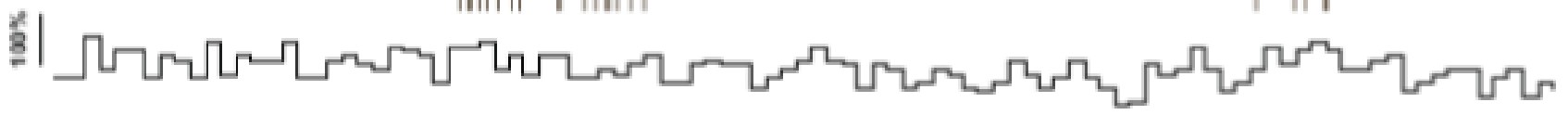
$\mid 2 \mathrm{~s}$

Fig. 4.

Representative spike rasters from populations of simultaneously recorded MSNs from WT and tgHD rats depicting instances of burst activity. Each horizontal raster represents activity from one MSN, providing $8 \mathrm{MSN}$ rasters for each genotype. The solid horizontal bars above each raster define bursts. Note that both WT and tgHD units display burst firing. WT bursting, however, is coordinated; thereby, engaging in coincident bursting among many unit pairs. Also note the somewhat elevated rate in WT rasters compared to tgHD. To illustrate the temporal structure of the population activity across the units, we calculated the global mean spike rate for all units in each raster. By calculating the mean \% difference in rate $(0.1 \mathrm{~ms}$ bins) for the $10 \mathrm{~s}$ period, we constructed a population activity histogram. It is evident by the large peaks and long valleys in the histogram that the activity in WT mice is more phasic and that bursts are coordinated across units compared to tgHDs. Note that the WT population activity histogram does not reflect the overall ongoing spontaneous activity; rather, it illustrates 
population activity during a discrete period of coincident bursting. This activity, moreover, is not apparent in tgHDs. 
a

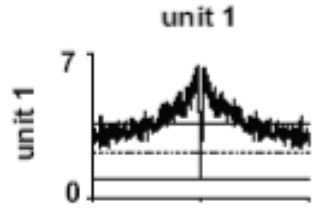

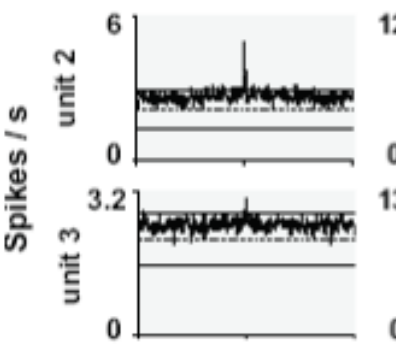
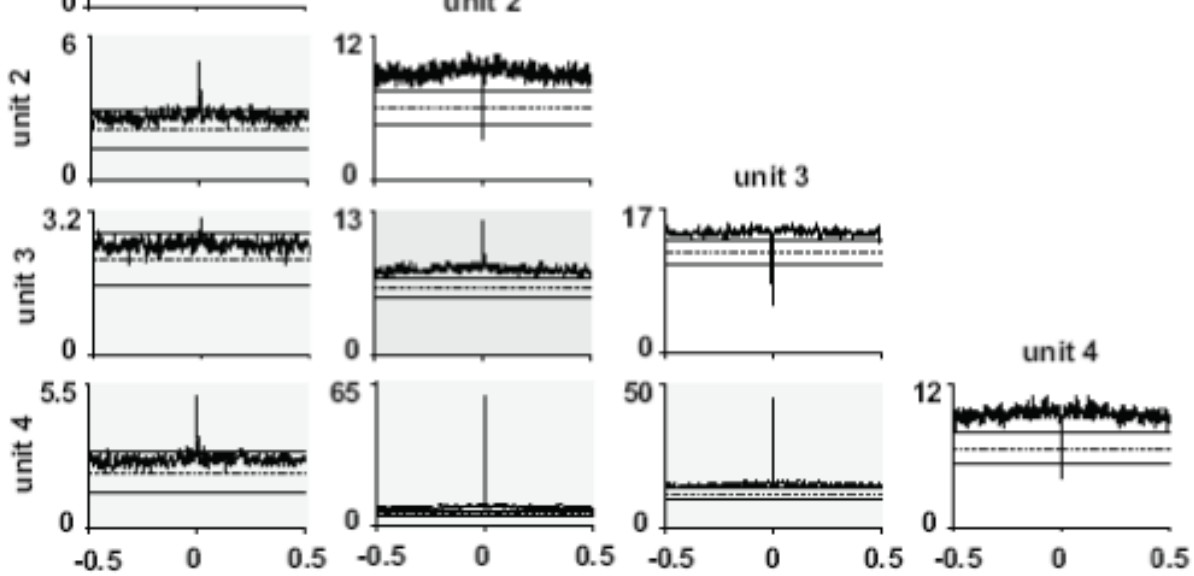

b

unit 1

$\operatorname{tg} H D$
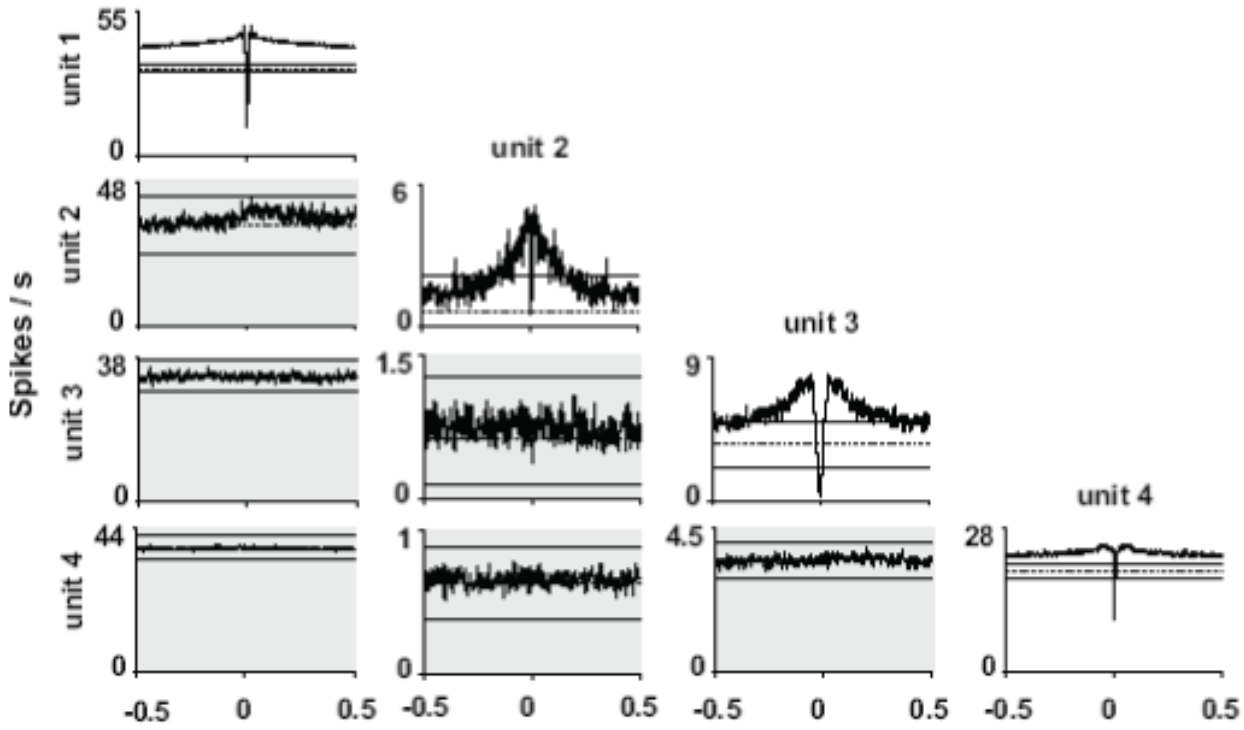

Time (s)

Fig. 5.

Representative cross-correlation histogram (CCHs) matrices constructed from simultaneous recordings of multiple MSNs in striatum of WT (a) and tgHD (b) rats over the entire $1 \mathrm{hr}$ recording session. The correlation matrices show all possible correlation pairs (bin =1 ms). The trigger units and the corresponding autocorrelation are in the top of each column and the reference units are in the left column. Gray shading indicates all CCHs (i.e., excluding autocorrelations). The horizontal solid bars in each $\mathrm{CCH}$ correspond to the upper and lower 99\% confidence intervals, and the horizontal dotted bar represents the expected mean firing rate. Note that autocorrelations are similar in WT and tgHD matrices. However, correlated unit pairs (indicated by central peaks that exceed the $99 \%$ confidence interval) dominate the WT 
matrix and are completely absent in the tgHD matrix. Overall, WTs had more correlated unit pairs than $\operatorname{tgHD}\left(\chi^{2}=30.1, P<0.001\right)$. Interestingly, the $\mathrm{CCH}$ for unit 1 vs. unit 2 in the tgHD matrix has a subtle, but non-significant peak. 
Table 1

Firing pattern of MSNs

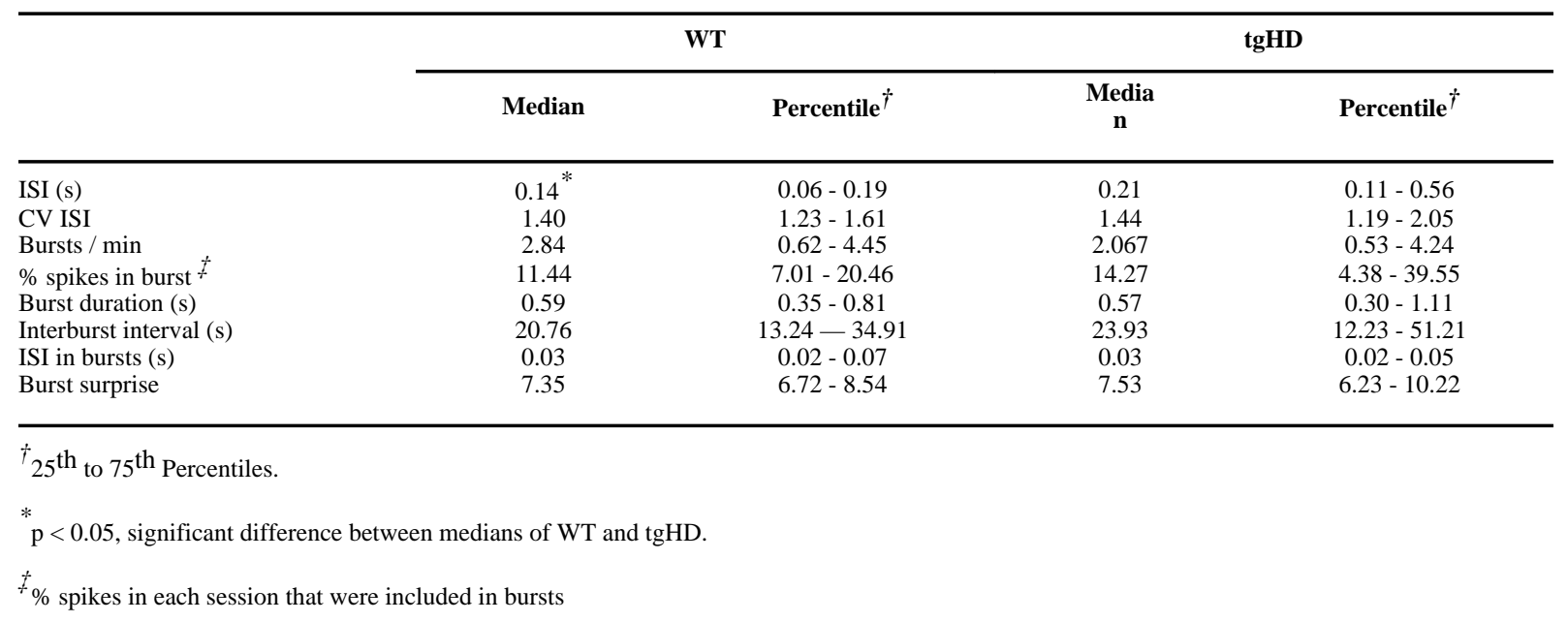

